\title{
Politique
}

Politique

\section{Alain Gagnon, et al., Les Opérations-Dignité : naissance d'un mouvement social dans l'est du Québec, Montréal, Éditions Léméac Inc., GRIDEQ et Carleton University Bookstore, 1981, 262 p.}

\section{Jean-Louis Gendron}

Volume 1, numéro 2, automne 1982

Les médias et les pouvoirs

URI : https://id.erudit.org/iderudit/040410ar

DOI : https://doi.org/10.7202/040410ar

Aller au sommaire du numéro

Éditeur(s)

Société québécoise de science politique

ISSN

0711-608X (numérique)

Découvrir la revue

Citer ce compte rendu

Gendron, J.-L. (1982). Compte rendu de [Alain Gagnon, et al., Les

Opérations-Dignité : naissance d'un mouvement social dans l'est du Québec,

Montréal, Éditions Léméac Inc., GRIDEQ et Carleton University Bookstore,

1981, 262 p.] Politique, 1(2), 179-181. https://doi.org/10.7202/040410ar d'utilisation que vous pouvez consulter en ligne.

https://apropos.erudit.org/fr/usagers/politique-dutilisation/ 
Alain Gagnon, et al., Les Opérations-Dignité: naissance d'un mouvement social dans l'est du Québec, Montréal, Éditions Leméac Inc., GRIDEQ et Carleton University Bookstore, 1981, 262 p. 
Tel que l'indique la présentation de ce volume, il s'agit là d'un recueil de textes cumulant les apports de disciplines fort variées à l'étude des mouvements sociaux en général et des Opérations-Dignité en particulier. Outre le directeur de l'ouvrage, Alain G. Gagnon, les personnes suivantes ont contribué à la parution de ce volume:

- Nick Baxter-Moore, «Mouvements sociaux, un cadre d'analyse théorique et comparatif».

- Léon Dion,

- Lionel Robert,

- Charles Banville,

«Émergence d'une conscience autodéterministe».

«Les Opérations-Dignité, à l'origine d'un mouvement populaire dans l'Est du Québec».

"L'origine et l'impact des Opérations-Dignité».

- Jean-Jacques Simard, «Les Opérations-Dignité: luttes d'espace et nostalgie ».

- Monique Dumais, «La dynamique d'un cri : une réflexion éthique sur les Opérations-dignité ».

- Claude Morin, «Éléments de synthèse».

Vues sous l'angle pluridisciplinaire de la sociologie, de la science politique, du journalisme, de l'éthique sociale, etc., les Opérations-Dignité font l'objet d'une analyse qui ne manque pas d'intérêt mais qui laisse parfois le lecteur sur son appétit.

En introduction, Nick Baxter-Moore brosse un tableau rapide de l'histoire, de la localisation et des impacts des Opérations-Dignité en tant que mouvements sociaux. Par la suite dans le premier chapitre, il élabore un cadre d'analyse théorique et comparatif. Ces deux textes sont de bons instruments d'analyse, mais on peut regretter que l'application du modèle aux Opérations-Dignité ne se fasse pas, par la suite, de façon plus rigoureuse et plus systématique. Tout au long de l'ouvrage, des 
jalons sont jetés pour l'analyse, mais cette analyse n'est pas suffisamment développée. C'est peut être là, une difficulté majeure inhérente à ce genre d'ouvrage collectif.

Ces articles, par ailleurs, sont pour la plupart très intéressants et même captivants à lire. Plusieurs essais d'interprétation, quoique encore plus impressionnistes que moulés sur le réel, n'en demeurent pas moins fort suggestifs. Ainsi, les chapitres préparés par Jean-Jacques Simard et par Monique Dumais sont particulièrement percutants, à cet égard.

Par contre, on peut regretter que l'excellent article de Léon Dion («Émergence d'une conscience autodéterministe») soit de nouveau reproduit ici, après avoir été publié une première fois dans la revue de l'ACELF (1971) et une deuxième fois dans La prochaine révolution (Leméac, 1973). Malgré la très grande pertinence qu'a eu cet article et la profondeur de pensée qui le caractérise, il eut mieux valu s'abstenir de cette répétition. Par contre, il eut été intéressant d'utiliser ce texte pour voir comment les espoirs et les intuitions de l'auteur se sont concrétisés ou pas, dix ans après une première publication.

En résumé, il s'agit donc très certainement d'un apport intéressant à l'étude des Opérations-Dignité et des mouvements sociaux, en général. On peut cependant regretter, comme nous l'avons mentionné, un certain manque de cohérence et d'unité dans l'analyse de même que le vieillissement de certains articles. On pourrait aussi déplorer une importance trop grande accordée aux visions prospectivistes, aux dépens d'un recours plus systématique aux données empiriques, ce qui aurait permis une analyse critique moins retenue des Opérations-Dignité ellesmêmes.

Jean-Louis Gendron, Université de Sherbrooke. 